\title{
Volume of Distribution Observed for Unbound Drug
}

National Cancer Institute

\section{Source}

National Cancer Institute. Volume of Distribution Observed for Unbound Drug. NCI

Thesaurus. Code C158265.

The volume of distribution associated with the terminal slope following administration, calculated using the observed value of the last non-zero concentration and corrected for unbound drug. 